\title{
Photoluminescent Si-based nanocrystals prepared by pulsed laser ablation in low-pressure helium-nitrogen mixtures for biomedical applications
}

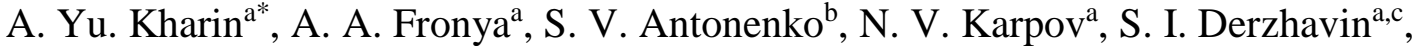 \\ Y. I. Dombrovska a, A. A. Garmash ${ }^{\mathrm{a}}$, N. I. Kargin ${ }^{\mathrm{b}}$, S. M. Klimentov ${ }^{\mathrm{a}}$, V. Yu. Timoshenko ${ }^{\mathrm{a}, \mathrm{d}}$, and \\ A.V. Kabashin ${ }^{\mathrm{a}, \mathrm{e}}$ \\ ${ }^{a}$ MEPHI, Institute of Engineering Physics for Biomedicine, Kashirskoe sh. 31, 115409 Moscow, \\ Russia; ${ }^{\mathrm{b}} \mathrm{MEPHI}$, Institute of Nanoengineering in Electronics, Spintronics and Photonics, \\ Kashirskoe sh. 31, 115409 Moscow, Russia; ${ }^{\mathrm{c}}$ Prokhorov General Physics Institute of the Russian \\ Acad. Sci., Vavilova St. 38, 117942 Moscow, Russia; ${ }^{\mathrm{d}}$ Lomonosov Moscow State University, \\ Physics Dep., Leninskie Gory 1, 119991 Moscow, Russia; ' Aix Marseille Univ., CNRS, LP3, \\ Campus de Luminy, Case 917, 13288 Marseille, France \\ *E-mail: s__asha@mail.ru
}

\begin{abstract}
Nanocrystalline silicon ( $\mathrm{Si}$ ) films were synthesized by nanosecond laser ablation of crystalline Si targets in low-pressure helium (He) and nitrogen $\left(\mathrm{N}_{2}\right)$ gas mixtures. Photoluminescence (PL) spectra of the prepared samples were found to depend on the $\mathrm{He} / \mathrm{N}_{2}$ ratio in the gas mixture. The ablation pure He atmosphere allowed us to prepare Si nanocrystals (NCs) exhibiting a PL band in red-near-IR range, while samples prepared in the presence of $\mathrm{N}_{2}$ exhibited a strong PL band with maximum in the green-yellow region. Such a modification of PL properties can be explained by the presence of amorphous $\mathrm{Si}$ oxynitride $\left(\mathrm{a}-\mathrm{SiN}_{\mathrm{x}} \mathrm{O}_{\mathrm{y}}\right.$ ) at the surface of $\mathrm{Si}$ nanocrystals. Structural studies of the prepared samples by means of the scanning electron microscopy revealed different morphology for Si-NCs produced under different gas mixtures. After treating of the films by ultrasound and dispersing in water, Si-NCs can be used as novel biodegradable markers for bioimaging, while the observed spectral tailoring effect makes possible an adjustment of the PL emission of such markers to a concrete bioimaging task.
\end{abstract}

Keywords: Silicon, nanoparticles, pulsed laser ablation in gas, films, photoluminescence

\section{INTRODUCTION}

Nanostructured silicon ( $\mathrm{Si}$ ) has attracted a lot of attention for last couple of decades due to a series of unique properties opening up avenues for diverse applications [1-3]. Biomedicine looks as one of main beneficiaries of these properties, as Si nanoparticles (NPs) are not only highly biocompatible [4] and biodegradable [5,6], but can also serve as contrast agents in bioimaging [7-9], sensitizers of various therapies under external stimuli [10-13] and carriers of anticancer drugs [5] and radionuclides [14].

Si nanocrystals (NCs) synthesized by methods of pulsed laser ablation present a viable alternative to nanostructures prepared by conventional chemical methods to offer required non-toxicity option for biomedical applications $[15,16]$. Such methods profit from a natural production of nanoclusters during the interaction of pulsed laser radiation with a target [17-19]. When ablated in a liquid ambient, the nanoclusters can be grown in a controllable manner to obtain solutions of colloidal NPs [20-22], which can be visualized by non-linear responses [23] and used as sensitizers in various therapies, including photodynamic therapy [20], radiofrequency radiation-induced hyperthermia [12] and photohyperthermia [24]. When ablated in gaseous ambience, the nanoclusters can be deposited on the target itself $[25,26]$ or on a separate substrate [27-29]. In contrast to Si-NPs prepared in liquid ambience, such nanostructures typically exhibit strong PL due to the exciton and defect-based emissions and can be used in imaging tasks. 
We recently developed a simple methodology based on pulsed laser deposition (PLD) in residual helium gas to fabricate Si-based nanocrystalline films [9]. In contrast to nanostructures prepared by laser pyrolysis of silane [7], such Si-NCs exhibited bright (quantum yield > 5\%) exciton-based PL around 750-850 nm even in the absence of wet chemistry-based step. We further managed to mill the laser ablated nanocrystalline films by ultrasound, disperse them in aqueous solutions and demonstrate their use as markers in bioimaging in cellular models [9]. Despite a great potential in bioimaging, their PLefficiency still needs amplification and tuning in order to match different spectral regions according to a concrete bioimaging task. We finally found possibilities for controllable tuning PL properties by performing PLD in mixtures of helium and nitrogen [30]. The addition of $\mathrm{N}_{2}$ gas led to the reduction of exciton band, while another bright band in the green-yellow range appeared. Such a tuning of PL looks promising for projected bioimaging applications.

Here, we provide details on structural and PL properties from Si NC films prepared under pulsed laser ablation in lowpressure helium-nitrogen mixtures and discuss their potential biomedical applications.

\section{EXPERIMENTAL METHODS}

In our experiments, a focused beam from a $\mathrm{KrF}$ excimer laser $(248 \mathrm{~nm})$ was used to irradiate a c-Si target at the angle of $45 \mathrm{deg}$. in the presence of He- $\mathrm{N}_{2}$ gas mixtures maintained at reduced pressures of 1-5 Torr. The target was constantly rotated in order to minimize the ablation of material from the same place on the target surface. A laser-initiated plasma plume expanded perpendicularly to the target surface and could be observed by a characteristic green emission associated with the PL of Si clusters in the vapor phase [17]. In this case, the presence of a buffer gas at reduced pressure enables one to cool down ablated nanoclusters in order to control the Si condensation and crystallization processes $[28,29]$. The nanoclusters were then deposited on a substrate (c-Si wafer or glass slide) placed $2 \mathrm{~cm}$ from the target. Such a deposition process led to the formation of Si-based nanostructured film after several thousands of laser pulses. The ablation was done under different proportions of $\mathrm{N}_{2}$ and $\mathrm{He}$ in gas mixtures.

A scanning electron microscope (SEM) system (TESCAN MAIA 3) operating at $0.1-30 \mathrm{kV}$ was used to study the morphology of laser-ablated films. Aqueous suspensions of Si NPs were prepared by $35 \mathrm{kHz}$ ultrasound treatment $(2$ $\mathrm{W} / \mathrm{cm}^{2}$ ) of laser-ablated films in an ultrasound bath for $30 \mathrm{~min}$. Dynamic light scattering (DLS) measurements of aqueous suspensions of Si-NPs were carried out by using a Malvern Zetasizer Nano ZS analyzer.
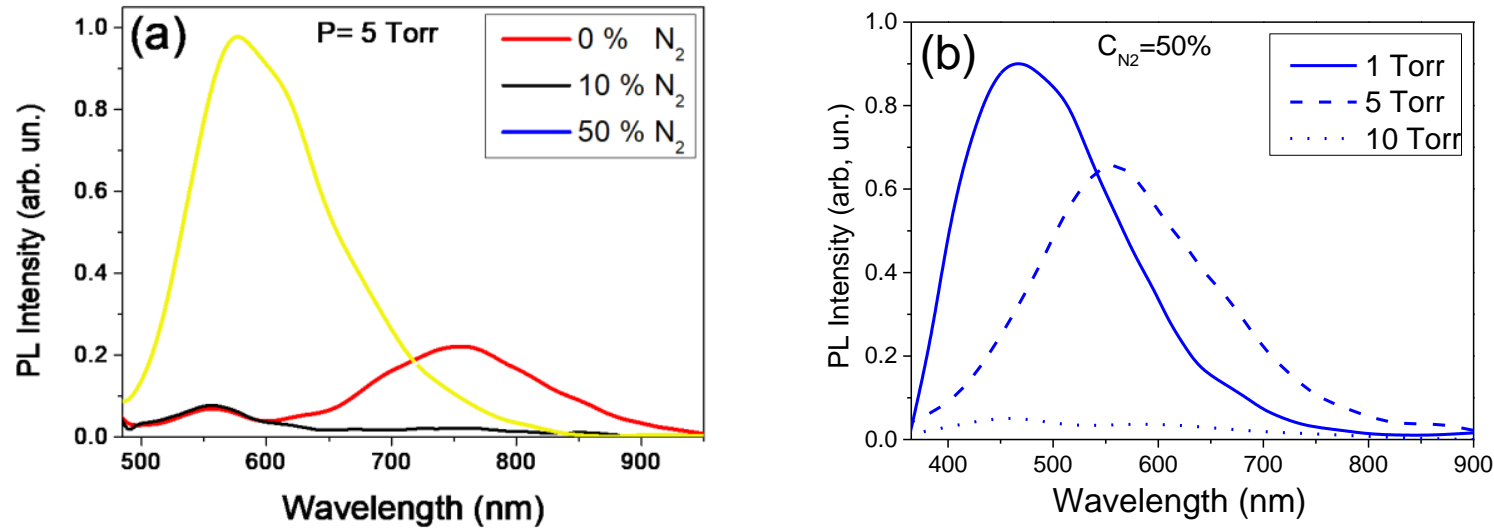

Figure 1. (a) PL spectra of laser-ablated Si films prepared in different gas mixtures with different $\mathrm{N}_{2}$ content under excitation at $450 \mathrm{~nm}$ and (b) PL spectra of films deposited at 1/1 ratio of gases under $350 \mathrm{~nm}$ excitation

\section{RESULTS AND DISCUSSION}

Laser-ablated films exhibited strong PL signals just after their exposition to ambient air, as shown in Fig. 1. One can see that PL spectrum for the films deposited in pure He (red curve in Fig. 1a) consisted of two PL bands centered around 760 $\mathrm{nm}$ and $550 \mathrm{~nm}$, respectively, which is consistent with results previous studies [9,28,29]. As we showed in Ref. [9], the first red band is due to the radiative exciton transitions in Si-NCs, while the second blue-shifted band can be explained by the radiative transitions via the localized electronic states in silicon suboxide $\left(\mathrm{SiO}_{\mathrm{x}}\right)$ coating of $\mathrm{Si}$ nanocrystals under the aging of laser-ablated films in ambient air [9,29]. The addition of a small (10\%) amount of $\mathrm{N}_{2}$ leads to immediate quenching of the exciton PL band, while the green band remains unaffected (black curve in Fig. 1a). However, a further 
increase of $\mathrm{N}_{2}$ content (blue curve in Fig.1a) leads to the evolution of the second band into at least 10-times more intense band in the yellow range (center at 570-580 nm). The generation of this green-yellow band can be unambiguously related to nitrogen-based passivation of $\mathrm{Si}$ nanocrystals and attributed to radiative transitions between electronic states in a$\mathrm{SiN}_{\mathrm{x}} \mathrm{O}_{\mathrm{y}}$ coating on the surface of S-NCs.

To further investigate PL properties of Si-NCs prepared by PLD, we fabricated three samples under the same ratio of gases in $\mathrm{He} / \mathrm{N}_{2}$ gas mixture (50/50), but at different absolute values of gas pressure (1 Torr, 5 Torr, 10 Torr). Fig. $1 \mathrm{~b}$ shows PL samples from these three samples under pulsed laser excitation at $351 \mathrm{~nm}$. One can see that the green-yellow band around 560-570 nm was recorded for the crystals deposited under moderate total gas pressure (5 Torr), while at high pressures (10 Torr) such a peak strongly decreased. On the other hand, S-NCs deposited at low gas pressures (1 Torr) exhibited a much blue-shifted PL peak around $460 \mathrm{~nm}$, which is typically attributed to defects in $\mathrm{SiO}_{2}$ centers $[9,29]$. These experiments evidence the presence of optimal pressure, which makes possible appropriate passivation of the nanocrystal surface in order to form a- $\mathrm{SiN}_{\mathrm{x}} \mathrm{O}_{\mathrm{y}}$ coating, capable of generating the green-yellow band.

To further clarify properties of Si-NCs prepared by PLD, we examined morphologies of films prepared under the same $\mathrm{He} / \mathrm{N}_{2}$ ratio, but at different total pressure. The SEM analysis shows that all deposited films exhibited nanograin textures, while the films deposited at low and high pressures had quite different structural morphologies. As shown in Fig. 2(a,b), in the case of relatively low pressures (1-5 Torr) the nanocrystals had relatively small visible size (5-10) and they combined together to form larger nanoparticle aggregates. On the other hand, the films deposited at higher pressure (10 Torr) contained much larger aggregations of Si-NCs (Fig. 2(c)). We believe that such a difference in morphologies of nanocrystalline films can be explained by different conditions of nanoclusters growth under different ambient pressures. Higher gas pressure leads to a faster condensation of Si nanoclusters under their collisions with gas species and a subsequent earlier crystallization before reaching the substrate. This leads to larger visible sizes of nanocrystal agglomerates deposited on the substrate. It should be noted that our earlier studies of the ablation in pure He atmosphere showed that the formed films are mainly composed of 2-4 nm Si-NCs embedded in amorphous silicon suboxide $(\mathrm{SiO}$, $0<x<1)$ matrix [29], while the mean grain size increase with the increase of He pressure.

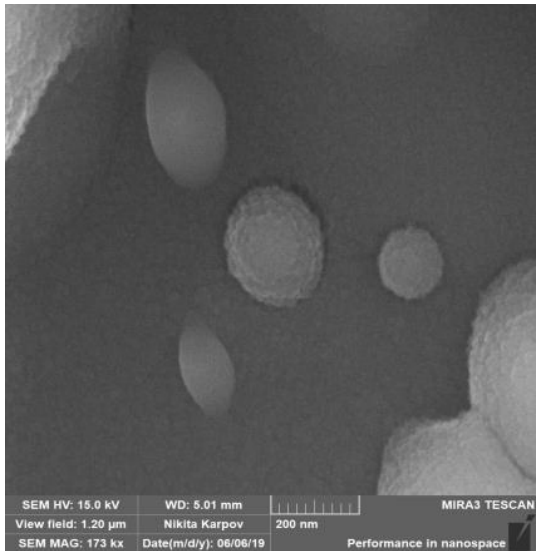

(a)

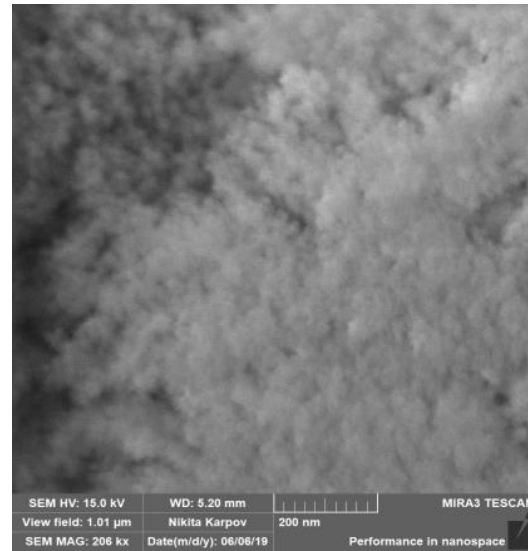

(b)

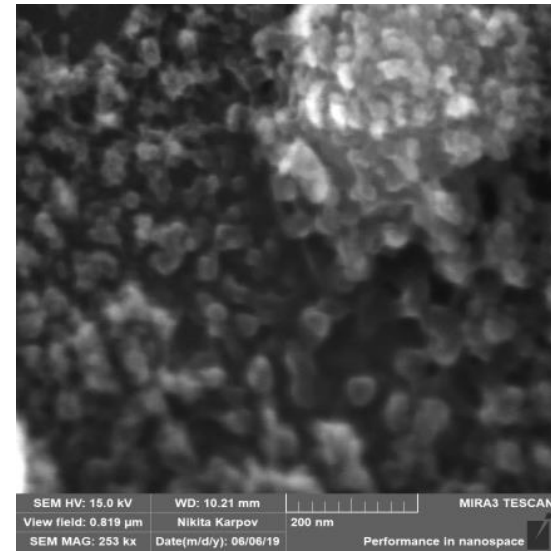

(c)

Figure 2. Top-view SEM images of Si-NC films obtained at 50\% $\mathrm{N}_{2}$ and different total pressures as 1 Torr (a), 5 Torr (b) and 10 Torr $(\mathrm{c})$.

To assess the properties of Si-NCs in aqueous media, which are essential for possible biomedical applications, we carried out ultrasound-assisted breakage (sonification) of the laser-deposited films. The ultrasonic treatment was performed in deionized water or saline $\left(0.9 \% \mathrm{NaCl}\right.$ in $\left.\mathrm{H}_{2} \mathrm{O}\right)$ for $1 \mathrm{~h}$. The power density and frequency of ultrasound were $5 \mathrm{~W} / \mathrm{cm}^{2}$ and $44 \mathrm{kHz}$, respectively. The laser-deposited films were detached from the substrate as a result of this sonification and dispersed in the solution forming a colloidal Si-NC based suspension. Fig. 3 shows DLS data on the hydrodynamic size of Si-NPs for samples prepared under different total pressures in 50/50 gas mixture. One can see that the hydrodynamic size increased with the increase of total gas pressure, which is consistent with results of SEM measurements (Fig. 2). It is important that for all gas pressures we were able to mill substrate-supported nanocrystalline films and disperse so-formed 
NPs in aqueous solutions. We earlier showed that such Si-based NPs, which consisted of Si-NCs as quantum dots, can serve as efficient markers for bioimaging [9].

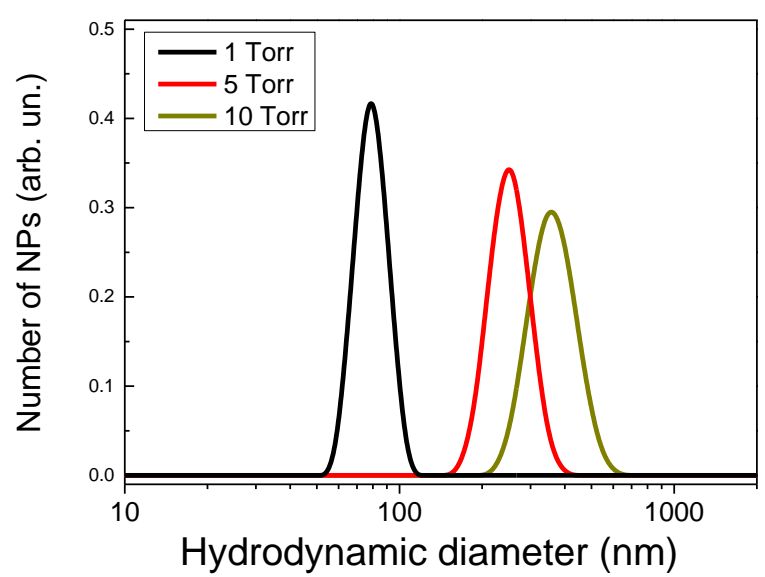

Figure 3. Hydrodynamic size distributions of Si NPs prepared from laser-ablated films obtained at $50 \% \mathrm{~N}_{2}$ at different total pressures.

The proposed methodology based on PLD from a Si target in helium/nitrogen mixtures, followed by ultrasound-based milling of the deposited nanocrystalline Si films, presents a promising pathway for the synthesis of brightly luminescent, water-dispersible and non-toxic Si-NPs. In the absence of wet chemistry step the Si quantum dots are exposed only to a clean environment (residual $\mathrm{He}$ and $\mathrm{N}_{2}$ gases, air, physiological solutions) and should have ultraclean surface. Combined with a possibility of sensitizing of various therapies under external stimuli [10-13] and carriers of drugs [14], lasersynthesised Si-NPs present a novel promising agent for cancer theranostics. On the other hand, the PLD technique makes possible the deposition of nanocrystalline porous Si films on an arbitrary substrate such as glass or metal surface, which can lead to novel applications. As an example, Si-based nanostructured films can be deposited on gold and be used as matrices for bioimmobilizations in surface plasmon resonance biosensors based on the Kretschmann-Raether geometry [31-33]. To excite surface plasmons over such a matrix, one has to use high refractive index silicon-based prism platform $[34,35]$.

\section{CONCLUSIONS}

By using methods of pulsed laser deposition in low-pressure helium $(\mathrm{He})$ and nitrogen $\left(\mathrm{N}_{2}\right)$ gas mixtures, we fabricated nanocrystalline $\mathrm{Si}$-based films exhibiting PL bands in the window of relative tissue transparency. We demonstrated the possibility of tuning the size of nanocrystallites and film morphology by changing operation pressure and gas ratio in the mixture. We also showed that the ablation pure He atmosphere leads to the formation of Si nanocrystal films exhibiting a PL band in red-near-IR range, while samples prepared in the presence of $\mathrm{N}_{2}$ exhibit a strong PL band with maximum in the green-yellow region. We finally demonstrated the possibility for milling of Si nanocrystals in aqueous solutions in order to obtain quantum dots, which can be used as markers in bioimaging applications.

\section{ACKNOWLEDGEMENTS}

The authors acknowledge the contribution from the Russian Science Foundation (Project 19-72-30012) for the fabrication and characterization of samples.

\section{REFERENCES}

[1] Hull, R. "Properties of Crystalline Silicon", Institution of Electrical Engineers: London (2006).

[2] Sailor, M. J. "Porous Silicon in Practice Preparation, Characterization and Applications", Wiley-VCH, Weinheim (2012).

[3] He, Y. "Silicon Nano-Biotechnology”, Springer: Heidelberg, (2014). 
[4] Canham, L. T. "Bioactive Silicon Structure Fabrication Through Nanoetching Techniques”, Adv. Mater. 7 (12), 1033-1037 (1995).

[5] Park, J.-H., Gu, L., von Maltzahn, G., Ruoslahti, E., Bhatia, S. N., Sailor, M. J. "Biodegradable Luminescent Porous Silicon Nanoparticles for in Vivo Applications", Nat. Mater. 8 (4), 331-336 (2009).

[6] Baati, T., Al-Kattan, A., Esteve, M. A., Njim, L., Ryabchikov, Y., Chaspoul, F., Hammami, M., Sentis, M., Kabashin, A. V., Braguer, D., "Ultrapure Laser-Synthesized Si-Based Nanomaterials for Biomedical Applications: In Vivo Assessment of Safety and Biodistribution”. Sci. Rep. 6, 25400 (2016).

[7] Erogbogbo, F., Yong, K.-T., Roy, I., Xu, G., Prasad, P. N., Swihart, M. T. "Biocompatible Luminescent Silicon Quantum Dots for Imaging of Cancer Cells". ACS Nano 2 (5), 873-878 (2008).

[8] Gu, L., Hall, D. J., Qin, Z., Anglin, E., Joo, J., Mooney, D. J., Howell, S. B., Sailor, M. J. “In Vivo Time-Gated Fluorescence Imaging with Biodegradable Luminescent Porous Silicon Nanoparticles”, Nat. Commun. 4, 2326 (2013).

[9] Gongalsky, M. B., Osminkina, L. A., Pereira, A., Manankov, A. A., Fedorenko, A. A., Vasiliev, A. N., Solovyev, V. V., Kudryavtsev, A. A., Sentis, M., Kabashin, A. V., Timoshenko, V. Yu. "Laser-Synthesized Oxide-Passivated Bright Si Quantum Dots for Bioimaging”, Sci. Rep. 6, 24732 (2016).

[10] Timoshenko, V. Y., Kudryavtsev, A. A., Osminkina, L. A., Vorontsov, A. S., Ryabchikov, Y. V., Belogorokhov, I. A., Kovalev, D., Kashkarov, P. K. "Silicon Nanocrystals as Photosensitizers of Active Oxygen for Biomedical Applications", JETP Lett. 83 (9), 423-426 (2006).

[11]Lee, C., Kim, H., Hong, C., Kim, M., Hong, S. S., Lee, D. H., Lee, W. I. "Porous Silicon as an Agent for Cancer Thermotherapy Based on near-Infrared Light Irradiation”, J. Mater. Chem.18 (40), 4790-4795 (2008).

[12] Tamarov, K. P., Osminkina, L. A., Zinovyev, S. V, Maximova, K. A., Kargina, J. V, Gongalsky, M. B., Ryabchikov, Y., Al-Kattan, A., Sviridov, A. P., Sentis, M., Kabashin, A. V., Timoshenko, V. Yu. "Radio Frequency Radiation-Induced Hyperthermia Using Si Nanoparticle-Based Sensitizers for Mild Cancer Therapy", Sci. Rep. 4, 7034 (2014).

[13] Sviridov, A. P., Andreev, V. G., Ivanova, E. M., Osminkina, L. A., Tamarov, K. P., Timoshenko, V. Y. "Porous Silicon Nanoparticles as Sensitizers for Ultrasonic Hyperthermia”, Appl. Phys. Lett. 103 (19), 193110 (2013).

[14] Petriev, V. M., Tischenko, V. K., Mikhailovskaya, A. A., Popov, A. A., Tselikov, G., Zelepukin, I., Deyev, S. M., Kaprin, A. D., Ivanov, S., Timoshenko, V. Y., Prasad, P. N., Zavestovskaya, I. N., Kabashin, A. V., "Nuclear Nanomedicine Using Si Nanoparticles as Safe and Effective Carriers of ${ }^{188}$ Re Radionuclide for Cancer Therapy", Sci. Rep., 2019, 9, (2017).

[15] Kabashin, A.V., Timoshenko, V. Y. "What Theranostic Applications Could Ultrapure Laser-Synthesized Si Nanoparticles Have in Cancer?" Nanomedicine 11, 2247-2250 (2016).

[16] Kabashin, A. V., Singh, A., Swihart, M. T., Zavestovskaya, I. N., Prasad, P. N. "Laser-Processed Nanosilicon: A Multifunctional Nanomaterial for Energy and Healthcare", ACS Nano 13, 9841-9867 (2019).

[17] Geohegan, D. B., Puretzky, A. A., Duscher, G., Pennycook, S. J., "Photoluminescence from Gas-Suspended $\mathrm{SiO}_{x}$ Nanoparticles Synthesized by Laser Ablation", Appl. Phys. Lett. 73, 438-440 (1998).

[18] Itina, T. E., Gouriet, K., Zhigilei, L. V., Noel, S., Hermann, J., Sentis, M. "Mechanisms of small clusters production by short and ultra-short laser ablation", Appl. Surf. Sci., 253, 7656-7661(2007).

[19] Itina, T. E. "On nanoparticle formation by laser ablation in liquids", J. Phys. Chem. C 115, 5044-5048 (2010).

[20] Rioux, D., Laferriere, M., Douplik, A., Shah, D., Lilge, L., Kabashin, A. V., Meunier, M. M., "Silicon Nanoparticles Produced by Femtosecond Laser Ablation in Water as Novel Contamination-Free Photosensitizers", J. Biomed. Opt. 14 (2), 021010 (2009).

[21] Intartaglia, R., Barchanski, A., Bagga, K., Genovese, A., Das, G., Wagener, P., Di Fabrizio, E., Diaspro, A., Brandi, F., Barcikowski, S. "Bioconjugated silicon quantum dots from one-step green synthesis", Nanoscale 4, 1271-1274 (2012).

[22] Al-Kattan, A., Ryabchikov, Y. V., Baati, T., Chirvony, V., Sánchez-Royo, J. F., Sentis, M., Braguer, D., Timoshenko, V. Y., Estève, M.-A., Kabashin, A. V. "Ultrapure Laser-Synthesized Si Nanoparticles with Variable Oxidation States for Biomedical Applications”, J. Mater. Chem. B 4, 7852-7858 (2016).

[23] Kharin, A. Y., Lysenko, V. V., Rogov, A., Ryabchikov, Y. V., Geloen, A., Tishchenko, I., Marty, O. Sennikov, P. G., Kornev, R. A., Zavestovskaya, I. N., Kabashin, A. V., Timoshenko, V. Y., "Bi-Modal Nonlinear Optical Contrast from Si Nanoparticles for Cancer Theranostics", Adv. Opt. Mater. 7, 1801728 (2019).

[24] Oleshchenko, V. A., Kharin A. Y., Alykova A. F., Karpukhina O. V., Karpov N. V., Popov A. A., Bezotosnyi V. V., Klimentov S. M., Zavestovskaya I. N., Kabashin A. V., Timoshenko V.Y. "Localized infrared radiation- 
induced hyperthermia sensitized by laser-ablated silicon nanoparticles for phototherapy applications" Appl. Surf. Sci. 145661 (2020).

[25] Kabashin, A. V, Meunier, M. "Visible Photoluminescence from Nanostructured Si-Based Layers Produced by Air Optical Breakdown on Silicon", Appl. Phys. Lett. 82, 1619-1621 (2003).

[26] Kabashin, A. V, Meunier, M. "Laser-Induced Treatment of Silicon in Air and Formation of Si/SiOx Photoluminescent Nanostructured Layers", Mater. Sci. Eng. B 101, 60-64 (2003).

[27] Patrone, L., Nelson, D., Safarov, V. I., Sentis, M., Marine, W., Giorgio, S. "Photoluminescence of Silicon Nanoclusters with Reduced Size Dispersion Produced by Laser Ablation”, J. Appl. Phys. 87, 3829-3837 (2000).

[28] Kabashin, A. V, Meunier, M., Leonelli, R. "Photoluminescence Characterization of Si-Based Nanostructured Films Produced by Pulsed Laser Ablation”, J. Vac. Sci. Technol. B. 19, 2217-2222 (2001).

[29] Kabashin, A. V, Sylvestre, J.-P., Patskovsky, S., Meunier, M. "Correlation between Photoluminescence Properties and Morphology of Laser-Ablated Si/SiO ${ }_{x}$ Nanostructured Films”, J. Appl. Phys. 91, 3248-3254 (2002).

[30] Fronya, A. A., Antonenko, S. V., Kharin, A. Y., Muratov, A. V., Aleschenko, Y. A., Derzhavin, S. I., Karpov N.V., Dombrovska Y.I., Garmash A.A., Kargin N.I., Klimentov, S. M., Timoshenko V. Y., Kabashin A.V. "Tailoring Photoluminescence from Si-Based Nanocrystals Prepared by Pulsed Laser Ablation in $\mathrm{He}-\mathrm{N}_{2}$ Gas Mixtures", Molecules, 25 (3), 440 (2020).

[31] Liedberg, B., Nylander, C. and Lunström, I., "Surface plasmon resonance for gas detection and biosensing," Sensor Actuat. B - Chem., 4, 299-304 (1983).

[32] Homola, J. "Surface plasmon resonance sensors for detection of chemical and biological species", Chem. Rev., 108(2), 462-93 (2008).

[33] Kabashin, A. V., Kochergin, V. E. and Nikitin, P. I., "Surface plasmon resonance bio- and chemical sensors with phase-polarisation contrast," Sensor Actuat. B - Chem. 54, 51-56 (1999).

[34] Patskovsky, S. et al, "Si-based surface plasmon resonance sensing with two surface plasmon polariton modes," Appl. Opt., 42, 6905 (2003).

[35] Patskovsky, S. et al, "Characterization of high-refractive index semiconductor films by Si-based Surface Plasmon Resonance”. Appl. Opt. 45, 6640-6645 (2006) 